\title{
Conflicto ambiental, transformaciones productivas y cambio institucional. Los comunales de Galicia (España) durante la transición a la democracia
}

Environmental conflict, productive transformations and institutional changes. The Galician commons (Spain) during the democratic transition DOI: 10.5935/2237-2717.20160006

\author{
David Soto Fernández \\ Laboratorio de Historia de los Agroecosistemas \\ Universidad Pablo de Olavide \\ dsotfer@upo.es \\ España
}

\section{RESUMEN}

El artículo se propone explorar la importancia de diferentes factores en los cambios en el conflicto ambiental en torno a los comunales. Analizaremos la importancia de las reglas (formales e informales) pero también la construcción de identidades por las comunidades locales en la interpretación de los comunales. Tenemos en cuenta, asimismo, los cambios en la funcionalidad ambiental y económica de los comunales. Nuestro estudio de caso se localiza en Galicia (noroeste de España). Nos centramos en un tipo de propiedad comunal no reconocida por ley hasta 1968, mostrando como la clarificación legal y la construcción de sistemas de reglas consistentes no es suficiente para explicar la sustentabilidad de los comunales. Los cambios en la funcionalidad de los comunales son también relevanmtes para entender su sustentabilidad en el largo plazo.

\section{PaLABRAS CLAVES}

Comunales, Conflicto Ambiental, Metabolismo Social.

\section{ABSTRACT}

In this paper our aim is to explore the importance of different factors in the explanation of changes in environmental conflicts in commons. We will analyze the importance of the rules (formal and informal) but also the making of identities by local communities regarding commons. We also explore the role of changes in the environmental and economic functionality of commons. Our case study is located in Galicia in NW Spain. We will analyze a particular example of commons not recognized by law until 1968, trying to show how the legal clarification and the construction of clear systems of rules are not sufficient to explain the sustainability of commons. Changes on functionality of commons are also relevant to understand the long term sustainability of the institution.

\section{KEYWORDS}

Commons, Environmental Conflict, Social Metabolism. 


\section{Introducción ${ }^{1}$}

Existen pocos aspectos del mundo rural de Galicia (NW de España) que hayan suscitado tanto interés académico como la propiedad comunal. Aunque la superficie comunal ha disminuido en la edad contemporánea lo ha hecho menos que en otras partes de España y aún goza hoy de gran importancia cuantitativa. De los 2,9 millones de hectáreas del territorio de Galicia, algo más de 670.000 hectáreas están registradas como propiedad colectiva. En una zona donde predomina la pequeña propiedad las tierras comunales son la única forma de gran explotación agraria (237 has de media), lo que las convierte en una institución de indudable interés para el desarrollo rural y para la aplicación de políticas agrarias. Durante la edad moderna y contemporánea los comunales jugaron un papel esencial en el equilibrio de las economías campesinas y en proporcionar la base de los cambios agrarios ligados a la primera revolución agrícola. El tipo de propiedad predominante de carácter germánico (propiedad privada pero colectiva sin distinción de cuotas) es además original en el contexto español. Por todo ello los comunales gallegos han sido objeto de atención desde ámbitos disciplinares dispares: la geografía, ${ }^{2}$ la Historia, ${ }^{3}$ la economía, ${ }^{4}$ la sociología, ${ }^{5}$ o la ciencia forestal. ${ }^{6}$ Asimismo se han desarrollado varios proyectos de carácter interdisciplinar donde la Historia ha jugado un papel central. ${ }^{7}$

Aunque estos trabajos han señalado las características positivas de los comunales de Galicia para el desarrollo rural también han destacado los problemas de los mismos. Muchas comunidades están institucionalmente inactivas o tienen una actividad precaria. Desde el punto de vista productivo muchos montes están infrautilizados y muchos proporcionan una renta exigua que no ofrece muchos estímulos a la acción colectiva. Desde el punto de vista medioambiental las tierras comunales de Galicia también tienen deficiencias. El mayor impacto en este sentido quizá sea el elevado número de incendios forestales presente de manera continuada desde hace cuatro décadas, aunque asimismo es de destacar la importancia de masas forestales en monocultivo y los consiguientes impactos en la biodiversidad. Las investigaciones realizadas en los últimos años también han destacado la existencia de numerosos ejemplos de comunidades con experiencias

\footnotetext{
${ }^{1}$ Este trabajo se ha beneficiado de la financiación siguiente: Proyecto "Sustainable Farm Systems: Long-Term SocioEcological Metabolism in Western Agriculture", financiado por el Social Sciences and Humanities Research Council, Canada. Proyecto "¿Sistemas agrarios sustentables? Una interpretación histórica de la agricultura española desde, la perspectiva biofísica", HAR2015-69620-C2-1-P, financiado por el Ministerio de Economía y Competitividad (España). El trabajo se ha realizado durante una estancia de tres meses en el Instituto de Ecología Social, Klagenfurt University, Viena financiado por el programa de movilidad "José Castillejo para jóvenes doctores 2014" (CAS 14/00039) del Ministerio de Educación, Cultura y Deporte (España).

${ }^{2}$ Abel Bouhier, Abel. La Galice. Essay geographique d'annalyse et d'interpretation d'un vieux complexe agraire. Imprimeirie Yonaisse, La Roche-Sur-Yon (Vendée), 1979, 2 vols.

${ }^{3}$ Xesús L. Balboa, (1990). O Monte en Galicia, Vigo, Xerais; Rico Boquete, Eduardo. (1995). Politica Forestal en Repoboacions en Galicia (1941-1971). Santiago de Compostela, Universidade de Santiago de Compostela; Soto, David. (2006). Historia dunha agricultura sustentábel. Transformacións productivas na agricultura galega contemporánea. Santiago de Compostela, Xunta de Galicia.

${ }^{4}$ Xaquín Fernández Leiceaga, Economía (política) do monte galego (Santiago: Universidade de Santiago de Compostela, 1990); María D. Domínguez García, y David Soto, "From an "integrated" to a "dismantled" landscape", in The Economic Value of Landscapes edited by C. Martijn van der Heide, Wim Heijman (London: Routledge, 2012), 204-223; Caballero, Gonzalo. (2014): "Community-based forest management institutions in the Galician communal forests: A new institutional approach". Forest Policy and Economics, DOI: 10.1016/j.forpol.2014.07.013.

${ }^{5}$ Lage, Xesús. Bosques, sociedad y cultura forestal en Galicia. (Vigo: Universidade de Vigo, 2003).

${ }^{6}$ Marey, M. and Rodríguez, V. "Forest transition in Northern Spain: Local responses on large-scale programmes of fieldafforestation." Land Use Policy 36-1 (2009): 139-156; Corbelle-Rico, E., Crecente-Maseda, R., Tubío-Sanchez, J. M. \& Onega-López, F. (2010). "Challenges in collective action for natural resource management: A study of common property regimes in the municipality of Guitiriz (northwest of Spain)." In Sustainable Use of Biological Diversity in Socio-ecological Production Landscapes. Background to the 'Satoyama Initiative for the Benefit of Biodiversity and Human Well-being, edited by C. Belair, K. Ichikawa, B. Y. L. Wong \& K. J. Mulongoy, 133-138 Canada: SCBD;

${ }^{7}$ Ana Cabana Iglesia, Ana Isabel García Arias, Marỉa do Mar Pérez Fra and Abel Rodríguez López. (2012). "El común de unos pocos, La infrautilización del monte vecinal en la montaña oriental gallega”, Ager, DOI: 10.4422/ager:2012.05.
} 
innovadoras de gestión y alternativas productivas originales que permiten afirmar que en algunos lugares se está produciendo una reinvención del comunal. ${ }^{8}$ Esta diversidad no puede ser adecuadamente explicada recurriendo a una sola tradición teórica (institucionalismo, economía ecológica, estudios culturales). En este trabajo pretendemos mostrar cómo sin la evolución sufrida por el comunal en el pasado reciente (especialmente en los últimos años del franquismo y la transición a la democracia) difícilmente se puede entender la situación actual. El caso de Galicia puede asimismo contribuir al debate sobre la pervivencia de los comunales ya que en él se entremezclan aspectos institucionales, productivos e identitarios. En el primer apartado del trabajo realizaremos un repaso a la literatura teórica sobre los comunales, tanto desde la perspectiva de la economía institucional como de la economía ecológica. En el segundo mostraremos las características actuales de los comunales de Galicia y los problemas que presentan, y en el tercero analizaremos los cambios históricos recientes que ayudan a entender la diversidad actual.

\section{Más allá de las instituciones. Reglas, condiciones materiales e identidad}

El estudio de los comunales se ha convertido en uno de los aspectos más discutidos en las Ciencias Sociales en las últimas décadas. Su análisis es relevante para entender problemas que interesan a disciplinas y tradiciones teóricas diferentes, especialmente para los interesados en la acción colectiva. ${ }^{9}$ Algunas de estas tradiciones y debates han influido el trabajo de los historiadores, pero sin duda, el debate central en toda la literatura ha sido el que hace referencia a la ineficiencia de los comunales a la hora de asegurar la sustentabilidad de los recursos naturales, partiendo de la tragedia de los comunes de Garret Harding ${ }^{10}$ y su argumentación de que los comunales propiciarían el agotamiento de los recursos al no existir restricciones al sobreaprovechamiento de los recursos. Una parte significativa del debate se ha centrado en la precisión terminológica y la distinción entre sistemas de propiedad comunal (Common property regimes) y recursos de uso común (common pool resources) así como entre estos y los bienes de acceso abierto (open access) o los club goods. El análisis institucional, que tiene su principal representante en Elinor Ostrom, ha sido quizás la tradición intelectual más influyente en el estudio de los comunales, centrando su agenda de investigación en el análisis de las reglas que explicarían el éxito o fracaso de las instituciones comunales. Para Ostrom ${ }^{11}$ muchas instituciones comunales desarrollaron históricamente complejos sistemas de autoorganización que potenciaron la cooperación y permitieron escapar a la tragedia del acceso abierto. El análisis institucional sería por tanto una herramienta poderosa para explicar la pervivencia de los comunales a lo largo del tiempo. El trabajo de Ostrom ha sido muy influyente en historiadores que se han preguntado por la organización y regulación de los comunales en distintos contextos históricos y por las lógicas de su pervivencia. ${ }^{12}$ Una aportación importante de la literatura historiográfica que ha dialogado con los

\footnotetext{
${ }^{8}$ Balboa et al., 2006; Cabana et al., 2012; Domínguez García, María Dolores., Swagemakers, P., Copena Rodríguez, D. , Covelo Alonso, J., Simón Fernández, X. (2014). "Collective agency and collaborative governance in managing the commons: the case of A Serra do Galiñeiro in Galicia, Spain", Spanish Journal of Rural Development in press.

${ }^{9}$ Un estado de la cuestión en Laerhoven, F. y Ostrom, E. "Traditions and Trends in the Study of the Commons", International Journal of the Commons, 1-1 (2007): 3-28.

${ }^{10} \mathrm{G}$. Harding, "The Tragedy of the Commons", Science 162 (1968): 1243-1248.

${ }^{11}$ E. Ostrom, "A General Framework for Analyzing Sustainability of Social-Ecological Systems", Science, 235 (2009):419422.

12 J. L. Van Zanden, "The paradox of the marks. The exploitaition of commons in the eastern Netherlands, 1250-1850", Agricultural History Review 47 (1999): 125-144; T. De Moor, "Avoiding tragedies: a Flemish common and its commoners under the pressure of social and economic changē during the eighteenth century", Economic History Review 62, (2009): 122.
} 
planteamientos de Ostrom ha sido situar al conflicto social en el centro de la explicación de la pervivencia y cambio de las regulaciones de las instituciones comunales. ${ }^{13}$

La otra gran tradición intelectual que ha influido el trabajo de los historiadores proviene de la Economía Ecológica ${ }^{14}$ y más recientemente por la implementación del aparato teóricometodológico del Metabolismo Social. ${ }^{15}$ Estas corrientes no desdeñan la importancia del entramado institucional ni de la producción de reglas que aseguren formas de manejo sustentables de los recursos, pero ponen el acento de la agenda de investigación en la parte material, en el estudio de los flujos biofísicos de energía y materiales entre y naturaleza y sociedad y también en los flujos de información que los regulan. El mundo físico no es considerado aquí un dato estático con el que interactúan las instituciones humanas, sino un agente activo de tal modo que las relaciones sociedad-naturaleza deben entenderse como un proceso de coevolución y de interacción mutua. En ese sentido se ha prestado atención a las diferentes formas de organizar el metabolismo social (caza recolección, agrario e industrial), a los perfiles metabólicos propios de cada una de estas formas y a los procesos de transición socio-ecológica entre unos y otros. ${ }^{16}$ En este contexto las formas de propiedad o de aprovechamiento comunal no son entendidas de manera ahistórica como sustentables o no sustentables sino si pueden contribuir a la sustentabilidad o no dependiendo de la organización del metabolismo social en la que están insertas. Esta tradición ha prestado, además, considerable atención al papel de los conflictos sociales en el mantenimiento o ruptura de manejos sostenibles de los recursos. El conflicto de nuevo juega un papel central en el mantenimiento de los comunales, pero también en los procesos de transición socioecológica que puedan hacer variar su sustentabilidad. ${ }^{17}$

Aunque ambas tradiciones ponen el acento en aspectos diferentes de la sustentabilidad de los bienes comunales no deben entenderse como opuestos e irreconciliables. De hecho en uno de sus últimos trabajos Elinor Ostrom planteaba un modelo de análisis de la sustentabilidad de los Sistemas Socio-Ecológicos que integra aspectos institucionales, físicos y sociales. ${ }^{18}$ Asimismo desde la Ecología política y la Historia Ambiental se ha planteado que los cambios operados en los comunales desde las revoluciones liberales se entienden mal si consideramos únicamente la tensión entre privado-público-comunal, es decir atendiendo únicamente a los derechos de propiedad. Martínez Alier ha propuesto el concepto de desarticulación de los comunales, para entender los cambios operados en los comunales desde el siglo XIX a partir de los cambios en la propiedad (privatización) pero también incluyendo las formas de manejo y funcionalidad de los mismos dentro de los agroecosistemas, así como la desarticulación social de las

\footnotetext{
${ }^{13} \mathrm{~J}$. M. Lana, "From equilibrium to equity. The survival of the commons in the Ebro Basin: Navarra from the 15th to the 20 th centuries", International Journal of the Commons 2.2 (2008): 162-191; P. Warde "Imposition, Emulation and Adaptation: Regulatory Regimes in the Commons of Early Modern Germany", Environment and History 19 (2013): 313-337; J. Lana y Laborda, M, "El anidamiento institucional y su dinámica histórica en comunidades rurales complejas. Dos estudios de caso (Navarra, siglos XIV-XX)", Documentos de Trabajo SEHA http://ideas.repec.org/p/seh/wpaper/1307.html.

${ }_{14}$ J. Martínez Alier, "Political Ecology, Distributional Conflicts and Economic Incomensurability" New Left Review 211 (1995): 70-88.

${ }^{15}$ M. González de Molina, y Toledo, V. Metabolismos, naturaleza e Historia. Hacia una teoría de las transiciones socioecológicas (Barcelona: Icaria, 2011).

${ }^{16}$ González de Molina, y Toledo, 2011.

${ }^{17}$ R. Guha, R. The unquiet wood: Ecological change and peasant resistance in the Himalaya, (New Delhi: Oxford University Press, 1989); J. Martínez Alier, El ecologismo de los pobres. Conflictos ambientales y lenguajes de valoración (Barcelona: Icaria, 2005); D. Soto, D.; Herrera, A.; González de Molina, M. y Ortega, A. "La protesta campesina como protesta ambiental, siglos XVIII-XX", Historia Agraria 42 (2007): 277-301.

18 Ostrom, 2009.
} 
comunidades que los gestionan. ${ }^{19}$ Este proceso ha sido estudiado por Antonio Ortega para la provincia de Granada en España entre los siglos XVIII y XX. ${ }^{20}$

Una de las aportaciones del trabajo de Ostrom más influyente en la literatura historiográfica ha sido su identificación de los famosos "principios de diseño", las características formativas básicas que explicarían el éxito y pervivencia en el largo plazo de instituciones comunales. Es su versión más reciente ${ }^{21}$ los ocho principios de diseño serían: La existencia de límites claros, tanto para los recursos como para los apropiadores de los mismos, reglas de apropiación y provisión congruentes entre sí y con las condiciones sociales y ambientales locales, canales de participación en la formación y modificación de las reglas, instrumentos de monitoreos de los recursos y de los apropiadores del mismo, escala graduada de sanciones, mecanismos de resolución de conflictos, reconocimiento de los derechos locales por el gobierno y sistemas de anidamiento institucional tanto vertical como horizontal. Como hemos señalado parte de la investigación histórica relevante ha tratado de proyectar hacia el pasado los planteamientos de Ostrom para explicar la pervivencia de los regímenes de propiedad comunal. ${ }^{22}$ Sin embargo como ha señalado Warde esta forma de plantear la cuestión peca de ahistoricidad ya que las instituciones comunales no existen en una especie de aislamiento histórico donde las condiciones cambiantes carecen de significación. ${ }^{23}$ En su trabajo Warde muestra como la formación de reglas de manejo de los comunales puede ser resultado de un complejo proceso de conflicto donde la imposición por poderes externos, la emulación de comunidades vecinas o la respuesta a las crisis, inciden en el diseño institucional. De hecho la propia Ostrom ha destacado en respuesta a sus críticos que la expresión "principios de diseño" no implica prescripción ni que esos principios estén en la mente de los creadores de sistemas comunales exitosos, y ha señalado que quizás un término mejor sería el de "buenas prácticas".

En realidad un examen más cuidadoso al instrumento general diseñado por Ostrom para el análisis institucional, ${ }^{24}$ muestra que aunque el análisis de las reglas juega un papel central, el mismo rango teórico de variables exógenas a cualquier situación de acción es ocupado por otros dos elementos, los atributos de la comunidad y las condiciones biofísicas y materiales. Este planteamiento permite no solo reconciliar la perspectiva institucional y la ambiental en el estudio histórico de los comunales, sino que introduce un tercer elemento mucho menos desarrollado por la literatura; ${ }^{25}$ el papel de la identidad de la comunidad y la construcción colectiva de objetivos y prioridades y la evaluación - de las experiencias. ${ }^{26}$ Paradójicamente esta cuestión ha sido mucho menos desarrollada por la historiografía a

\footnotetext{
${ }^{19}$ Martínez Alier, J., "Pobreza y Medio Ambiente. A propósito del Informe Brundtland” in La Tierra: Mitos, Ritos y Realidades editado por González de Molina, M. and González Alcantud, J.A. (Granada: Anthropos/Diputación Provincial de Granada 1992), 295-332.

${ }^{20}$ Ortega, A. La tragedia de los cerramientos. La desarticulación de la comunalidad en la provincia de Granada, (Valencia: Fundaçión Instituto de Historia Social, 2002).

${ }^{21}$ E. Ostrom, "Beyond Markets and States: Polycentric Governance of Complex Economic Systems", American Economic Review 100 (2010): 641-672.

${ }^{22}$ Especialmente interesante resulta en mi opinión el ejercicio realizado por Laborda y Lana (2013) de aplicar el concepto de anidamiento institucional a la evolución histórica de los comunales navarros.

${ }^{23}$ Warde, P. "Imposition, Emulation and Adaptation: Regulatory Regimes in the Commons of Early Modern Germany", Environment and History 19 (2013): 313-337

${ }^{24}$ E. Ostrom, Comprender la diversidad institucional (Oviedo: KRK, 2013).

${ }^{25}$ En el libro de Ostrom este aspecto ocupa tan solo un epígrafe a pesar de que tiene la misma jerarquía teórica que las otras dos variables.

${ }^{26}$ D. Gallego, "Las distintas caras de la economía institucional", XIII Congreso de la SEHA, Badajoz (2013).
} 
pesar del enorme desarrollo de la Historia Cultural en los últimos años. ${ }^{27}$ Desde nuestro punto de vista la adecuada comprensión de las transformaciones históricas de los comunales debería atender tanto al conjunto de normas que los regulan (tanto formales como informales) como las condiciones biofísicas y materiales (y que entre otras cosas nos indican lo que es posible hacer y lo que no es posible hacer de ninguna manera en un contexto concreto) como a la construcción de la identidad colectiva (que entre otras cosas explica las diferencias entre lo que una u otra sociedad entiende como racionalidad).

Pero comunidades, reglas y condiciones biofísicas y materiales interrelacionan en contextos históricos que son potencialmente conflictivos. De hecho una parte relevante de la literatura señala que el conflicto es un elemento explicativo central en la emergencia de instituciones para el manejo de los recursos de uso común. Por ejemplo para McCay ${ }^{28}$ no es la preocupación por el agotamiento o degradación del recurso lo que explicaría la emergencia de las instituciones comunales, sino el conflicto por el acceso a los recursos, lo que coincide con los planteamientos de Paul Warde enunciados más arriba. Estos planteamientos concuerdan asimismo bien con la extensa teoría existente sobre conflictividad ambiental, y especialmente con la idea de un environmentalism of the poor realizada por Joan Martínez Alier y Ramachandra Guha. ${ }^{29}$ Según estos autores existen luchas ecologistas tanto actuales como en el pasado en comunidades que, independientemente de poseer o no una ideología ecologista, llevan a cabo una defensa del acceso y distribución igualitaria de los recursos naturales. De acuerdo con esta idea los conflictos sobre los recursos de uso común tanto en la actualidad como en el pasado serían una variante de los conflicto ecológico-distributivos. Aunque coincidimos con la idea de que los conflictos sobre los recursos son conflictos ambientales, independientemente de si son conceptualizados como tales por las comunidades implicadas, no con la idea de que el acceso y la distribución sean la única característica relevante para evaluar el papel de un conflicto en la sustentabilidad. En otro lugar ${ }^{30}$ hemos señalado que aquellos conflictos en los que, además del acceso y la distribüción, está en juego un cambio en las formas de manejo de los recursos son más relevantes en términos de sustentabilidad. ${ }^{31}$ En esos casos el resultado del conflicto no afectará tan sólo a la cantidad del recurso que se apropia, ni a los grupos que se lo apropian, sino también a la manera en que el recurso es apropiado (conflictos reproductivos). Por ejemplo en el caso de que el resultado de un conflicto sobre unas superficies comunales cambie un manejo agro-silvo-pastoril campesino por un manejo intensivo industrial. La hipótesis que queremos desarrollar en este artículo es que son precisamente este tipo de conflictos los que están presentes en el proceso de desarticulación de los comunales que se produce en muchos lugares desde finales del siglo XVIII y han influido decisivamente en la transformación de las lógicas de las instituciones comunales. A partir del estudio de caso de los comunales de Galicia pretendemos mostrar como los cambios en la comunidad, las condiciones biofísicas y las regulaciones han

\footnotetext{
${ }^{27}$ Una notable excepción en Izquierdo, J. El rostro de la comunidad. La identidad del campesino en la Castilla del antiguo régimen (Madrid: CES, 2002).

${ }^{28}$ B. McCay, B. "Emergence of Institutions for the Commons: Contexts, Situations, and Events", in The Drama of the Commons edited by Ostrom, E., Dietz, T., Dolsak, N., Stern, P. C., Stonich, S. and Weber, E. U.,( Washington D.C.: National Academy Press, 2002), 361-402.

${ }^{29}$ Guha, 1989; Guha, R.; Martínez-Alier, J. Varieties of environmentalism. Essays north and south (London, Earthscan, 1997); Martínez Alier, 2005.

${ }^{30}$ D. Soto, D.; Herrera, A.; González de Molina, M. y Ortega, A., 2007, 277-301.

${ }^{31}$ En el citado trabajo se realiza una distinción conceptual entre conflictos ambientales (aquellos donde solo está en cuestión el acceso o distribución del recursos), ambientalistas (donde además del acceso y la distribución están en cuestión también las formas de manejo) y ecologistas (donde además existe un lenguaje ecologista explícito). Ver asimismo González de Molina, M, Soto Fernándeż, D., Garrido, F. Los conflictos ambientales como conflictos sociales. Una mirada desde la ecología política y la historia. Ecología Política, 50 (2016), pp. 31-38.
} 
influido en la aparición de conflictos y a su vez han sido modificadas por los resultados de los mismos.

\section{Los comunales gallegos en la actualidad}

La zona de estudio que hemos escogido para desarrollar en este trabajo presenta características inusuales en el contexto español. Galicia, la región noroeste del país no se corresponde con las características reconocibles para la mayor parte del Estado. Región de clima atlántico, agricultura de pequeña explotación campesina y con una creciente especialización ganadera durante los siglos XIX y XX muestra entre sus características específicas una importancia notable de los montes, una parte considerable bajo regímenes de propiedad comunal hasta la actualidad. La mayoría de los montes de Galicia pertenecían a un tipo específico de sistema de propiedad, los Montes Vecinales en Mano Común (MVMC) que son los que nos interesan aquí. Hasta la revolución liberal en Galicia los municipios eran muy escasos por lo que la presencia de montes de propiedad municipal lo era también. La titularidad de los MVMC estaba asignada a la vecindad en el territorio (mayoritariamente una parroquia) al que pertenecía el monte. Normalmente se les define como montes de propiedad privada pero colectiva donde el derecho de propiedad se obtenía con la vecindad y se perdía en caso de perder la misma. En términos institucionales la titularidad pertenecía colectivamente a la comunidad campesina de manera imprescriptible e inembargable.

La historia contemporánea de la propiedad vecinal en Galicia es una Historia conflictiva y paradójica. A finales del Antiguo régimen la mayoría de las tierras no cultivas de la región (cerca de dos millones de hectáreas sobre un total de 2,9 millones) correspondían a este tipo de propiedad. ${ }^{32}$ Después de la Revolución Liberal la propiedad vecinal (privada pero colectiva) desaparecerá de la legislación al ser equiparada a la propiedad municipal (pública), situación que se mantendrá desde 1812 hasta 1968. La falta de reconocimiento legal no implicó que los comunales dejaran de gestionarse por las comunidades de vecinos (hasta la dictadura de Franco), pero favoreció que muchas comunidades campesinas repartieran e individualizaran los comunales para prevenir la desposesión. ${ }^{33}$ Ello explica que en la actualidad solo una parte de los montes gallegos sobrevivan como montes de vecinos. Una de las paradojas de la historia de los comunales gallegos es que está situación comenzó a revertirse durante el periodo donde la intervención estatal -en los montes fue más intensa (el franquismo). En 1968 se aprobó la primera ley que reconocía y regulaba la existencia de la propiedad vecinal (Ley de Montes Vecinales en Mano Común de 27-VI1968). ${ }^{34}$ La explicación de esta paradoja será uno de los objetivos de este trabajo y está relacionada con la intensa conflictividad campesina sobre los comunales. Durante los quince años posteriores a la ley de 1968 la mayoría de los montes serán devueltos a las comunidades, en un proceso asimismo conflictivo, de tal manera que en la actualidad cerca de 2800 montes están reconocidos como montes vecinales. Esta historia de desposesión y de conflicto, junto con los cambios productivos en la agricultura gallega, han marcado la arquitectura institucional de los comunales, que no responden ya a la misma lógica productiva que en el pasado, ni se organizan de la misma manera ni siquiera son

\footnotetext{
${ }^{32}$ Bouhier, 1979 P. Saavedra, "O que non se pode medir: Os recursos do comunal nas economías campesiñas de Galicia de 1600 a 1850", Actas do Congreso de Montes Veciñais, 14-16 de Decembro de 1995, Xunta de Galicia, Santiago.

${ }^{33}$ L. Fernández Prieto, y Soto, D. "El Atlántico no es El Mediterráneo. El cambio agrario al otro extremo de la Península Ibérica: El mismo Estado, otros paisajes, ¿Los mismos campesinos?, in Ramón Garrabou. Sombras del progreso. Las huellas de la Historia Agraria edited by Ricardo Robredo (Barcelona: Crítica, 2010), 231-264.

${ }_{34}$ Durante la etapa democrática se aprobará una segunda ley estatal en 1980 y otra de carácter autonómico en 1989).
} 
entendidos de la misma forma por unas comunidades que asimismo han cambiado sus características definitorias.

Pero, ¿Cuál es la situación actual de las tierras vecinales en Galicia y en qué difiere con el pasado? Disponemos de un estudio interdisciplinar, realizado por historiadores y economistas, que elaboró una base de datos con información para el año 2000 para la totalidad de los montes de vecinos y un estudio en detalle de tres comarcas representativas. ${ }^{35}$ Asimismo disponemos de trabajos más recientes sobre estudios de caso localizados. ${ }^{36}$ Existían a comienzos del presente siglo 2835 comunidades que ocupaban 673.681 hectáreas (el $23 \%$ de la superficie total). La mayoría de los comunales se concentran en el sur de la región (provincias de Pontevedra y Ourense), mientras que en el norte la mayoría de la superficie es de propiedad privada individual (figura 1). De acuerdo con las estimaciones del trabajo citado 150.000 casas tenían derechos de propiedad lo que supone un total de población implicada en la propiedad vecinal de 530.000 personas (un $20 \%$ del censo de población). Estos datos son suficientes para demostrar la relevancia de los comunales para el desarrollo rural en Galicia. Pero por debajo de estos datos generales nos encontramos con una enorme diversidad productiva y de gestión en los comunales. Aunque la actividad principal en la actualidad es la forestal nos encontramos con actividades económicas variadas (ganadería, energía eólica, usos recreativos, canteras) y con comunales muy activos y económicamente rentables junto con otros infrautilizados o abandonados.

Figura 1 - Distribución de los montes de vecinos en Galicia en la actualidad (\%)

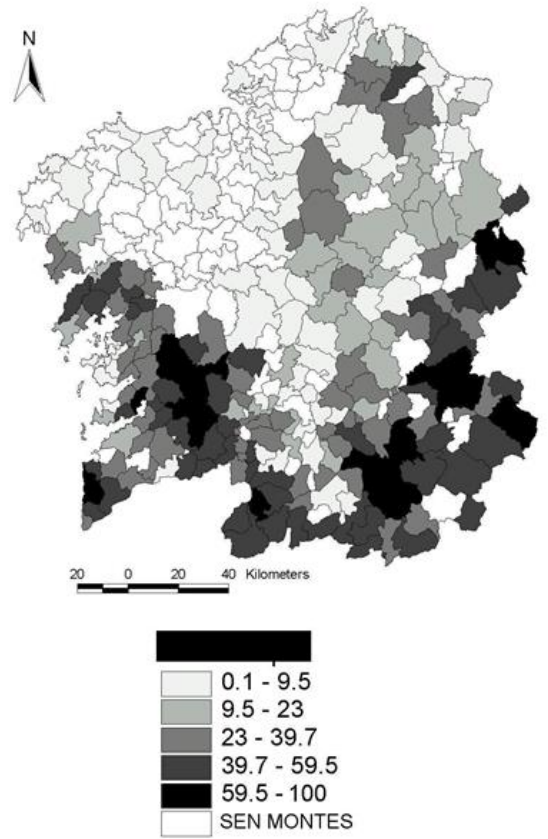

Fuente: Balboa et al, 2006

\footnotetext{
${ }^{35}$ La comarca de O Condado en la provincia de Pontevedra, la comarca de Sarria en la provincia de Lugo y la comarca de Baixa Limia en la provincia de Ourense. Balboa López, X.; Besteiro Rodríguez, B.; Fernández Leiceaga, X.; Fernández Prieto, L.; Jordán Rodríguez, M.; López Iglesias, E.; Soto Fernández, D.; Viso Outeiriño, P. Os montes veciñais en man común: o patrimonio silente. Naturaleza, economía, identidade e democracia na Galicia rural (Vigo, Xerais, 2006).

${ }^{36}$ A. Cabana Iglesia, Ana I. García Arias, María do Mar Pérez Fra y Abel Rodríguez López. "El común de unos pocos, La infrautilización del monte vecinal en la montaña oriental gallega", Ager (2012), DOI: 10.4422/ager.2012.05; Domínguez García, María Dolores., Swagemakers, P., Copena Rodríguez, D. , Covelo Alonso, J., Simón Fernández, X. (2014). "Collective agency and collaborative governance in managing the commons: the case of A Serra do Galiñeiro in Galicia, Spain", Spanish Journal of Rural Development in press.
} 
Desde el punto de vista de la gestión nos encontramos con comunidades abandonadas o con escasa actividad por parte de los propietarios y comunidades con mucha participación así como comunales gestionados directamente por las comunidades y otros gestionados por la administración forestal autonómica mediante convenio con los propietarios. El $64 \%$ de las comunidades y el $76 \%$ de la superficie tienen algún tipo de aprovechamiento, aunque el $25 \%$ de las comunidades y el $42 \%$ de la superficie tienen sus aprovechamientos gestionados indirectamente por la administración forestal. Un porcentaje significativo (un $36 \%$ de las comunidades y un $24 \%$ de la superficie) estaba abandonado en el año 2000. Estos casos son de zonas en proceso de despoblación acelerada y suponen uno de los principales problemas en la gestión de los montes tanto por su infrautilización económica como por los problemas de vulnerabilidad ambiental que generan. Nos encontramos por tanto que tan sólo un 38\% de las comunidades estaban gestionadas directamente por los propietarios. Esto está directamente relacionado con la estructura demográfica. Tan sólo un 21\% de ellos eran en el año 2000 activos en el sector agrario por un $47 \%$ de activos en otros sectores y un $32 \%$ de jubilados. El mayor porcentaje de gestión directa se da en aquellas zonas donde la mayoría de la población trabaja en la industria o en el sector servicios. ${ }^{37}$ Esto nos está indicando que la mayoría de los comunales con implicación de los vecinos no están relacionados con la actividad agraria (con la que estaban históricamente relacionados los montes) sino con nuevas formas de entender la funcionalidad de estos (beneficios comunitarios, espacios recreativos, medioambiente sano). Un indicador del dinamismo de estas comunidades (presentes especialmente en la Galicia occidental) lo tenemos en la presencia en estas zonas de conflictividad vinculada tanto con la gestión y el acceso como con los aprovechamientos de los comunales. ${ }^{38}$ Las comunidades más activas tienden a ser mayoritariamente urbanas o periurbanas y con una presencia significativa de propietarios ligados a actividades urbanas y no rurales.

Desde el punto de vista ambiental nada ejemplifica mejor los problemas de los bosques gallegos (tanto los de propiedad privada individual como los vecinales) que el impacto de los incendios forestales (figura 2). Desde comienzos de la década de los setenta este ha sido un problema recurrente ha sido objeto de debate social y político y que ha ocupado buena parte del esfuerzo investigador. ${ }^{39}$ Más allá del debate sobre las causas y los responsables de los incendios, no hay duda de que es un problema relativamente reciente (que no está presente antes de la década de los sesenta) y que está relacionado con la desarticulación de los espacios forestales, la segregación de los usos del suelo y en definitiva con la ruptura de los equilibrios en el manejo del paisaje. En tanto que una buena parte de la superficie quemada fue y sigue siendo de propiedad colectiva interesa comprender cuales son las lógicas que funcionan detrás de los comunales bien organizados y con implicación de las comunidades, ya que estas son-los más resilientes frente a los incendios.

\footnotetext{
${ }^{37}$ Balboa et al, 2006.

${ }^{38}$ Balboa et al, 2006.

${ }^{39} \mathrm{G}$. Serrano, Gonzalo. El régimen de propiedad y los incendios forestales en Galicia. (Santiago de Compostela:

Universidade de Santiago de Compostela; Lage), 2003.
} 
Figura 2 - Superficie afectada por incendios en Galicia

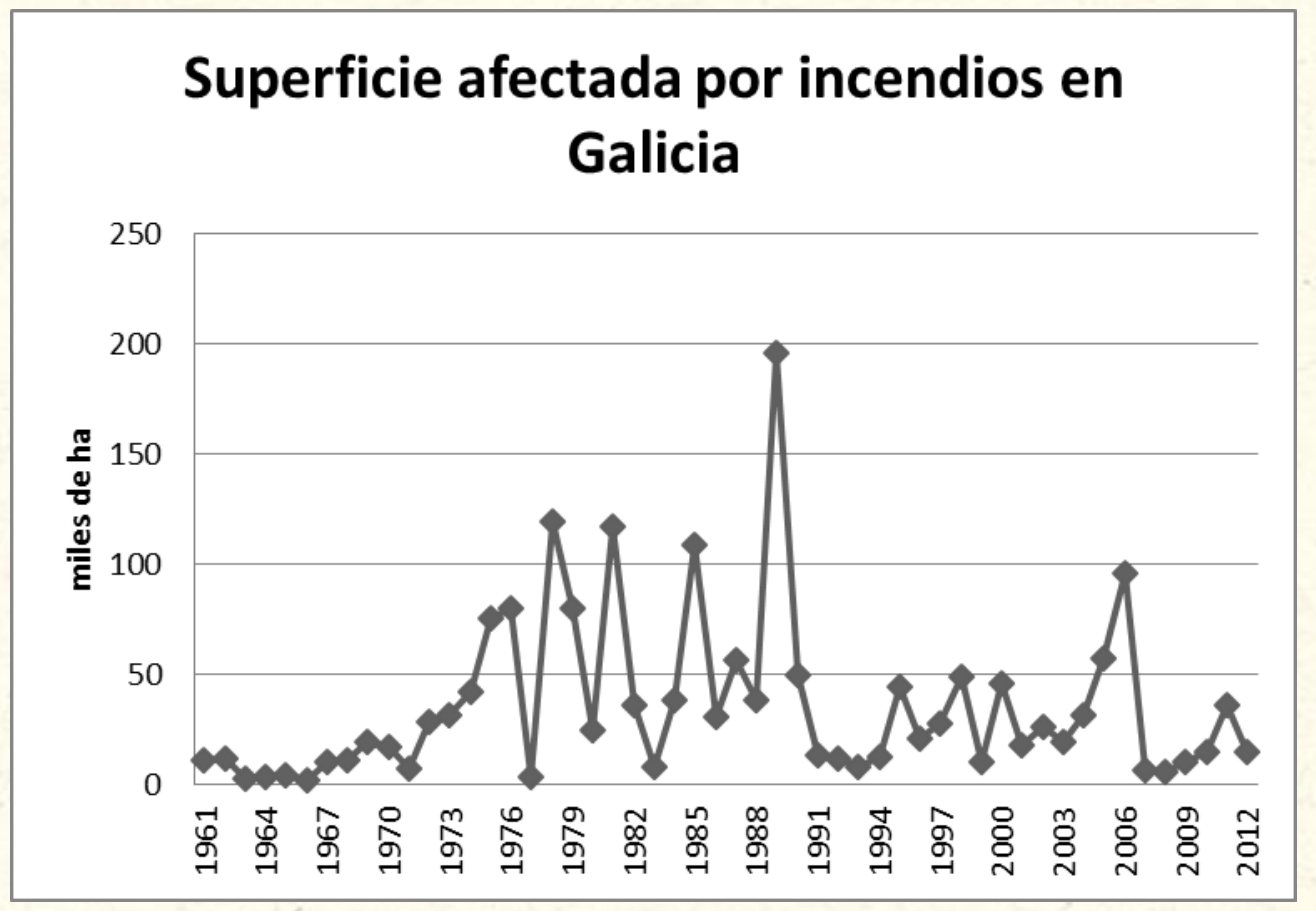

Fuente: Anuarios de estadística Agraria e INE.

Pensamos que los planteamientos teóricos de Ostrom, y especialmente los principios de diseño pueden ser un punto de partida interesante para aproximarse a la problemática de la propiedad vecinal en la Galicia de hoy, aunque en este caso es importante distinguir entre la letra de la ley y la aplicación real de la misma así como tener en cuenta la complejidad de situaciones. Desde el punto de vista legal ${ }^{40}$ no hay duda de que la propiedad vecinal cumple prácticamente todos los principios enumerados por Ostrom. Se establecen límites claros para los recursos (superficie legalmente reconocida como vecinal) como para los propietarios (vecindad), reglas claras que establecen límites para la apropiación, canales de participación, instrumentos legales de control e incluso instituciones supracomunitarias tanto creadas por las propias comunidades (asociaciones de comunidades de vecinos) como por la administración. Desde el punto de vista de sistema de derechos de propiedad las comunidades de vecinos poseen cuatro de los cinco establecidos por: El derecho de acceso, el derecho de obtención del recurso, el derecho de manejo y el derecho de exclusión. Únicamente el derecho de alienación está fuertemente limitado por el carácter imprescriptible e indivisible de la propiedad. Pero en la práctica nos encontramos con numerosos ejemplos de comunidades que actúan al borde de la ley o directamente al margen de esta, tanto en lo que respecta al acceso al recurso como en lo que respecta a los derechos de propiedad. Para entender esto es imprescindible analizar cómo es interpretado el comunal hoy que en gran medida difiere de cómo era interpretado en el pasado. Este cambio está relacionado con los cambios productivos sucedidos en las últimas décadas, pero también por la lógica de la propia ley que, queriendo recrear el comunal tradicional, en realidad lleva a cabo una recreación de un comunal imaginado que no existió nunca en esa forma. Esto no es necesariamente un problema para aquellas comunidades que responden razonablemente a las nuevas lógicas de entender el comunal, pero sí lo puede ser para

\footnotetext{
${ }^{40}$ Balboa et al, 2006; G. Caballero "Community-bäsed forest management institutions in the Galician communal forests: A new institutional approach". Forest Policy and Economics (2014). DOI: 10.1016/j.forpol.2014.07.013.
} 
aquellas que aun responden a las viejas lógicas mal adaptadas a la realidad productiva actual y al carácter marcado por la ley.

La vieja comunidad, que con matices mantiene sus características esenciales hasta mediados del siglo XX difiere en muchos aspectos de la comunidad actual, pero quizás el elemento más importante de diferencia sea que su principal funcionalidad era el mantenimiento del equilibrio campesino más que la consecución de la equidad. ${ }^{41}$ Las superficies vecinales jugaban un papel productivo fundamental en como sostén del sistema agrario tradicional, ${ }^{42}$ pero también los cambios en la intensidad de los usos del monte permiten entender las condiciones del crecimiento agrario vivido por la agricultura gallega desde mediados del siglo XVIII hasta la guerra civil. ${ }^{43}$ Además de su importancia productiva no se puede minimizar su papel como definidor de la identidad local. Es la titularidad colectiva del monte la que se convierte en muchos casos en elemento constitutivo de la vecindad. El vecino es el copropietario del monte y este elemento puede legar a ser más significativo que la pertenencia a una parroquia o a un municipio. ${ }^{44}$ Las tierras comunales cohesionan socialmente a la comunidad local. Uno de los aspectos centrales de esta es el equilibrio entre casa y colectivo, ejemplificado en la titularidad común y en aprovechamiento familiar (que no estrictamente individual) de los montes. Tres características básicas por tanto definen a la vieja comunidad. En primer lugar el aprovechamiento doméstico de los recursos colectivos se explica por la necesidad que existe del monte para mantener la productividad agro-ganadera. En segundo lugar el aprovechamiento no era ni se entendía como igualitario. Aunque la titularidad es ejercida por todos los vecinos de manera indiferenciada y el disfrute jurídicamente igualitario el aprovechamiento es mayor en aquellas casas con más tierras, ganado y fuerza de trabajo. De la misma manera y en tercer lugar aunque la gestión era colectiva, tampoco era igualitaria. La desigualdad existente afectaba a la toma de decisiones. Ello no quiere decir que la titularidad colectiva no jugase un papel corrector de la desigualdad en las comunidades rurales. Aunque el acceso no es igualitário, - los comunales sí juegan un papel esencial en el mantenimiento de los sectores más desfavorecidos de la sociedad siendo, por tanto, estas instituciones un instrumento importante de equidad.

Algunos de los elementos característicos de esta vieja comunidad permanecen presentes en la actualidad, pero fundamentalmente entre los comuneros más mayores, mayoritariamente jubilados y con escasa capacidad de gestión sobre el monte. No se puede decir por tanto que la vieja comunidad haya desaparecido, pero sí que está en retroceso frente a nuevas formas de entender la comunidad que han sido favorecidas además por la legislación. Aunque todas las leyes desde 1968 reconocen y legalizan los aprovechamientos individuales indudablemente la comunidad recreada por la ley de 1968 y especialmente por las siguientes parte de una concepción claramente igualitaria en el acceso y en los aprovechamientos, así como en una gestión democrática favorecida por el hecho de que el proceso de devolución de la propiedad vecinal coincidió con el proceso de transición a la democracia y en muchos sitios los conflictos por ambos procesos transcurrieron

\footnotetext{
${ }^{41}$ Xesús Balboa, (1990). O Monte en Galicia, Vigo, Xerais. Una característica similar es observada para Navarra por Lana (2008).

${ }^{42}$ Bouhier, 1979.

${ }^{43}$ Tres son los recursos básicos extraídos del comunal: alimentos para el ganado, cosechas suplementarias de cereal y, especialmente fertilizante. Una descripción de estos usos y de su evolución contemporánea en Soto (2006; 2015).

${ }^{44}$ Una diferencia importante entre la vieja y la nueva comunidad se encuentra aquí precisamente. Los derechos de propiedad se otorgan por la vecindad y se pierden con esta, pero esto no estaba pensado para la llegada de inmigrantes ajenos al lugar (algo muy raro) sino para hacer sitio a la apertura de nuevas casas de habitantes del lugar. Esto hoy puede en ocasiones acarrear conflictos con la llegada dē inmigrantes a zonas en proceso de despoblación (Balboa et al, 2006; Cabana et al, 2012).
} 
paralelamente. ${ }^{45}$ Estos cambios tienen que ver evidentemente con las transformaciones en los usos (del uso agro-ganadero del monte al uso mayoritariamente forestal). La percepción colectiva es además negativa hacia el reparto de beneficios monetarios entre los vecinos. De manera mayoritaria las comunidades reinvierten los beneficios en la gestión del propio recurso o en la realización de obras públicas o comunitarias. En el estudio realizado sobre tres comarcas representativas encontramos que tan sólo un $6 \%$ de las comunidades repartían beneficios monetarios entre los vecinos y algo similar sucede en la actualidad de acuerdo con otros estudios de caso como en la comarca de Vigo. ${ }^{46}$ El comunal es entendido en estos casos como un recurso esencial proveedor de servicios, en ocasiones sustitutivo de una gestión municipal que no siempre atiende suficientemente a las parroquias rurales. Aunque el comunal es privado, y es percibido claramente como privado, su funcionalidad se asemeja más a un bien público que a un recurso de uso individual. Las comunidades que responden a esta nueva forma de entender las superficies vecinales se corresponden bastante bien con las comunidades más activas y con una gestión propia de los recursos. Nos encontramos en buena medida con una ruptura entre la vieja y la nueva comunidad que se observa además claramente en la forma de percibir la situación de las tierras comunales. De manera mayoritaria los vecinos propietarios de mayor edad consideran que los comunales están peor aprovechados en la actualidad que antes de la devolución de la propiedad y son partidarios de volver a los usos tradicionales de carácter individual (aunque no realizados por ellos mismos sino por los más jóvenes) mientras que los vecinos más jóvenes por el contrario son partidarios de esta nueva manera de entender la comunidad y consideran que los montes se manejan mejor hoy. Desde el punto de vista de la identidad la situación reciente muestra una situación de transición entre la vieja concepción de la comunidad y la nueva, aunque es evidentemente arriesgado asegurar el predominio futuro de la nueva, ya que para muchas comunidades el problema fundamental es la mera supervivencia de la misma por razones demográficas. ${ }^{47}$

\section{De la Antigua a la nueva comunidad. Cambios productivos y conflictos por la propiedad durante el franquismo y la transición a la democracia}

La principal explicación del paso de la vieja a la nueva forma de entender la comunidad radica en el largo y conflictivo proceso de devolución de la propiedad a los vecinos que sucedió en un momento histórico en el que se produjeron dos transformảciones de envergadura en la sociedad española y gallega: en primer lugar el cambio político con la transición de la dictadura a la democracia y en segundo el cambio productivo ligado al proceso de industrialización de la agricultura y la ruptura de los equilibrios agro-silvopastoriles anteriores. Aunque desde el análisis institucional se ha afirmado que la devolución de la propiedad a los vecinos y el consiguiente proceso de cambio institucional se produjo de manera progresiva y gradual lo cierto es que existe un elevado grado de consenso en la historiografía sobre el carácter conflictivo y rupturista del proceso. ${ }^{48}$ Todos estos trabajos

\footnotetext{
${ }^{45}$ Xesús Balboa, Besteiro Rodríguez, B.; Fernández Leiceaga, X.; Fernández Prieto, L.; Jordán Rodríguez, M.; López Iglesias, E.; Soto Fernández, D.; Viso Outeiriño, P. "La devolución de la propiedad vecinal en Galicia (1960-1985). Modos de uso y conflicto de propiedad", Historia Agraria 33 (2004): 105-130.

${ }^{46} \mathrm{M}$. Domínguez García et al. "Collective agency and collaborative governance in managing the commons: the case of $A$ Serra do Galiñeiro in Galicia, Spain", Spanish Journal of Rural Development 2014 in press.

${ }^{47}$ Ana Cabana Iglesia et al., 2012, 5.

${ }^{48}$ Rico, 1995; Rico Boquete, E. "Política forestal y conflictividad social en el noroeste de España durante el primer franquismo, 1939-1959", Historia Social (2000) 38: 117-140; Fernández Prieto y Soto, 2004; Balboa et al, 2004; Cabana Iglesia, A. "Minar la paz social. Retrato de la conflictividad rural en Galicia durante el primer franquismo", Ayer 61 (2006), 267-288.; A. Freire, "El proceso de devolución de los montes vecinales en mano común: una historia de lucha social por los recursos naturales (1968-1989)" in Por surcos y cālles. Movilización social e identidades en Galicia y País Vasco (19681980) edited by Daniel lanero (Madrid: La catarata, 2013), 66-107.
} 
coinciden en señalar también que el proceso arranca con la conflictividad desatada contra el proceso de repoblación forestal y de segregación de usos impulsado por la dictadura franquista. Esta conflictividad no evitó la crisis de la agricultura orgánica campesina, pero si puso en peligro el proceso de repoblación forestal desarrollado por el régimen.

Figura 3

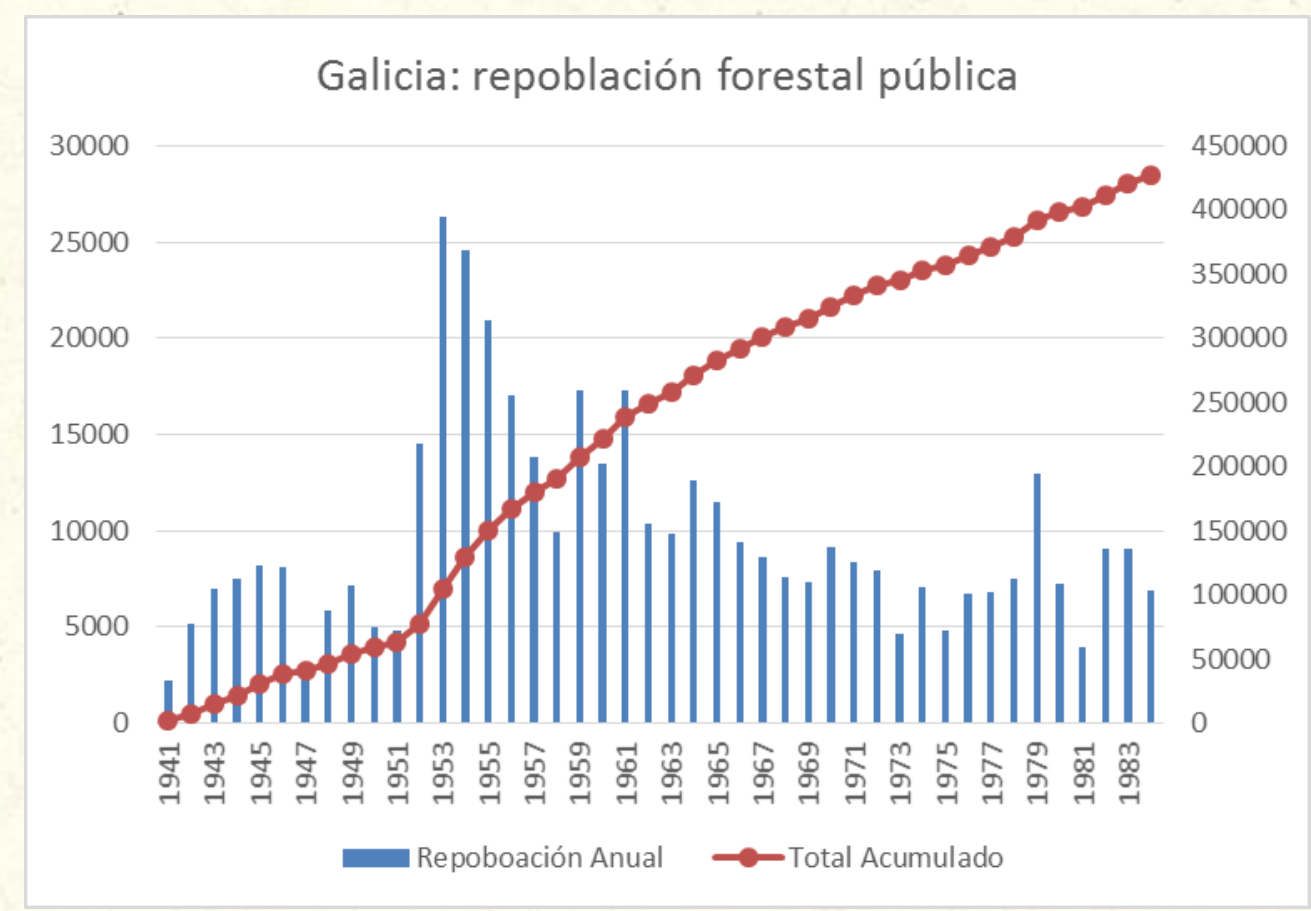

Fuente: Anuarios de estadística Agraria

A lo largo de las dos décadas en que la política autárquica es la principal matriz de la economía franquista se hará imposible el manejo del territorio integrado, propio de la agricultura gallega anterior a la guerra civil, en el que el equilibrio de los usos agrícolas, ganaderos y forestales era un elemento central del funcionamiento del agroecosistema. El régimen impulsará un proceso de desposesión de los comunales y de creación de consorcios con los ayuntamientos que provocará la prohibición y exclusión de los usos campesinos. De un monte multifuncional se irá pasando a un concepto de bosque en monocultivo e incompatible con los usos agro-ganaderos. A la altura de 1964 los servicios forestales del Estado habían consorciado ya 475.000 hectáreas de montes en Galicia, la inmensa mayoría de ellos vecinales, y se habían repoblado ya más de 270.000 hectáreas por parte de los servicios forestales del Estado (Figura 3). El modelo de silvicultura intensiva era manifiestamente incompatible con el manejo multifuncional campesino. Pero a pesar del contexto dictatorial represivo, la repoblación provocó una considerable protesta de las comunidades rurales que llevó a hacer pensar a la administración forestal del régimen que la política de repoblación estaba en peligro. El uso forestal se convirtió para muchas comunidades campesinas en el enemigo, por lo que fueron muy variadas las fórmulas para oponerse a la repoblación, incluyendo mecanismos legales y de resistencia abierta, así como también se utilizó el recurso al incendio como mecanismo de resistencia. La amplia distribución de estos conflictos a lo largo del territorio gallego es especialmente significativa al producirse en un contexto dictatorial. Este proceso arroja luz sobre varias cuestiones relevantes en la comprensión del mantenimiento de las instituciones comunales. En primer lugar porque en cierto sentido el conflicto tendrá éxito y el franquismo se verá obligado a 
reconocer la titularidad de los montes a través de la ley de 1968. Pero al mismo tiempo este éxito se producirá en un contexto de profundos cambios sociales y económicos que mudarán las características de la comunidad campesina y la propia funcionalidad de los montes vecinales.

Figura 4

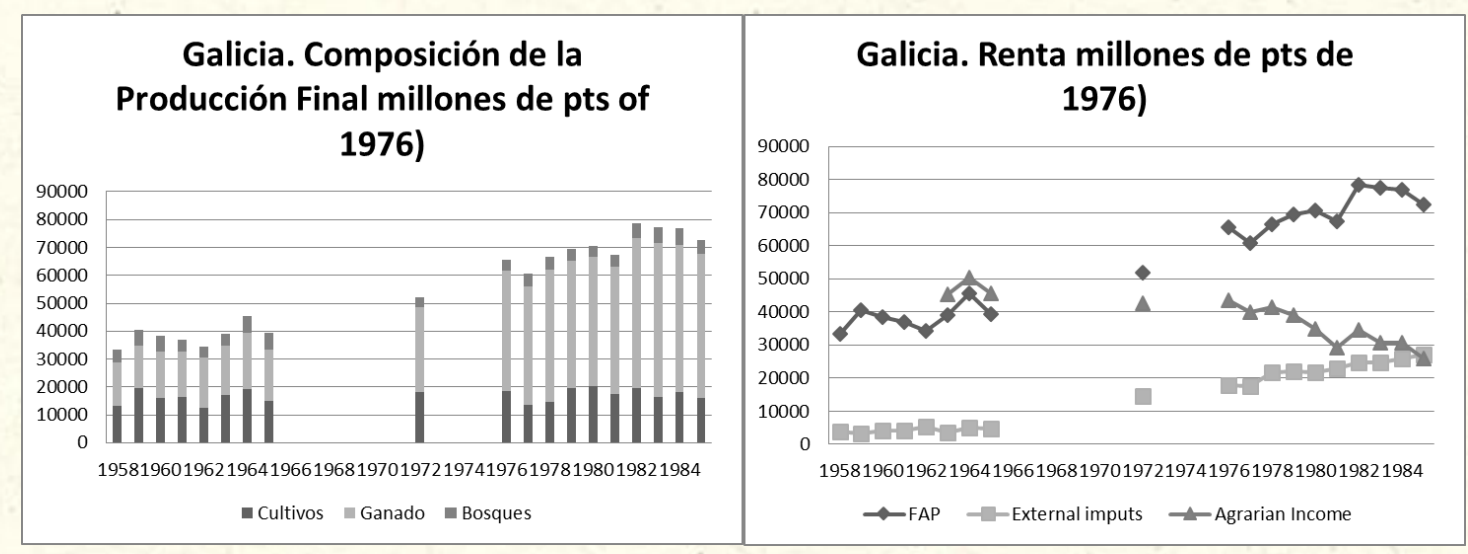

Fuente: Soto, 2006

La comunidad campesina que protesta por la repoblación forestal mantiene las mismas características que durante periodos anteriores, y en este sentido se reivindica el papel tradicional del monte en las economías campesinas, pero entre las décadas de los sesenta y setenta se producirán grandes cambios entre los que podemos citar de manera significativa la emigración, desagrarización y desarticulación de muchas comunidades, pero también la industrialización de la agricultura y la especialización comercial en ganadería de vacuno de leche. Esto llevará a que el conflicto se plantee en los sesenta no en relación al mantenimiento de los usos campesinos frente al uso forestal sino entre uso forestal y uso ganadero del monte (vía creación de pastizales). En la figura 4 se recogen las características económicas principales del proceso de transformación de la agricultura. Junto con un crecimiento relevante, aunque moderado, de la Producción Final Agraria nos encontramos con un proceso de especialización ganadera acelerada lo que se observa en el crecimiento de su participación en la PFA (y con un peso muy escaso de la parte forestal). Este proceso de especialización va unido asimismo a un proceso de industrialización de la agricultura que a lo largo de la década de los setenta se va a ir haciendo progresivamente más dependiente económicamente de inputs externos que de los reempleos. Este primer elemento de cambio es relevante para la adecuada comprensión de los cambios en la propiedad vecinal. Permite explicar que amplios sectores de la sociedad gallega, y lo que es más importante algunos sectores políticamente relevantes del régimen, favorezcan y promuevan la devolución de la propiedad a las comunidades vecinales. El objetivo en este caso no será ya la defensa de los manejos campesinos sino promover el uso ganadero enfrentándose a los servicios forestales para los que la devolución de la propiedad solo era un instrumento para hacer viable socialmente el aprovechamiento forestal. ${ }^{49}$

\footnotetext{
${ }^{49}$ Balboa et al. 2004.
} 
Figura 5 - Origen de la alimentación animal en Galicia.

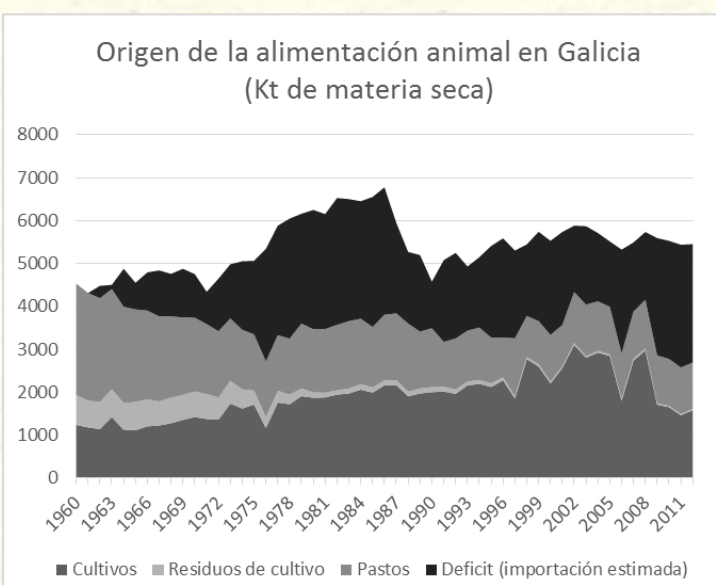

Origen de la alimentación animal en \%

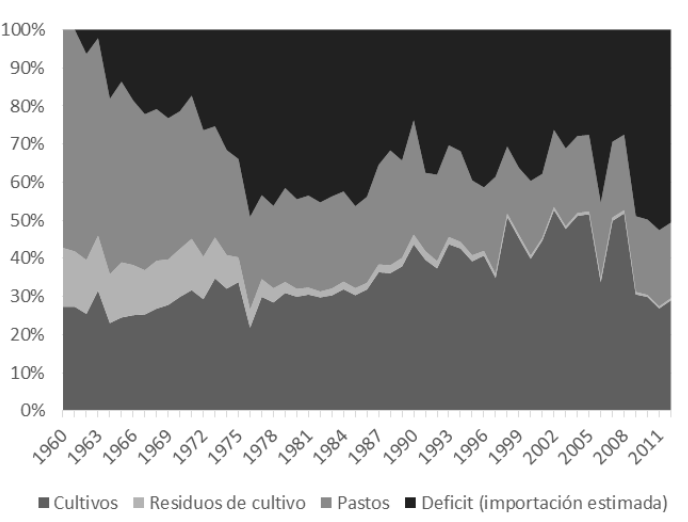

Fuente: Soto Fernández, D. Del manejo multifuncional del territorio a la desarticulación productiva: cambios en los flujos de biomasa durante el proceso de industrialización de la agricultura gallega (1960-2012). Documentos de Trabajo SEHA, DT-SEHA 1505 (2015).

Pero el proceso de industrialización de la agricultura gallega trajo consigo, al igual que en el conjunto de España, una caída muy significativa de la renta agraria en términos absolutos (figura 4). Esta caída está ligada a la caída a su vez de los precios, a la reducción de la población activa agraria (en términos per cápita por tanto esta caída debe ser matizada) y, en el caso gallego a una fragmentación de las explotaciones entre las que consiguen redimensionarse y adaptarse a las nuevas necesidades de la agricultura (especialmente en la mitad norte de la región) y una mayoría de explotaciones que acaban derivando hacia la agricultura a tiempo parcial o a la desaparición vía envejecimiento. Esta fragmentación socioeconómica que arranca del proceso de industrialización agraria tiene mucha incidencia en la diversidad productiva y organizativa presente en las comunidades desde la devolución de la propiedad. Pero al mismo tiempo una pregunta permanece abierta y es la razón por la que por lo menos aquellas comunidades con presencia de ganadería rentable no recurrieron desde la devolución de la propiedad a los comunales para abaratar el coste en alimentación. De hecho, y a pesar de algunos programas públicos en este sentido la creación de pastizales ha sido muy minoritaria desde la década de.los. La explicación de esto radica en la propia dinámica del proceso de industrialización y de su progresiva dependencia de los mercados. De hecho, y aunque en un principio la explicación de la especialización ganadera está relacionada con las ventajas comparativas del territorio, a medida que avanza la industrialización se ha ido produciendo una desarticulación entre las explotaciones ganaderas y el territorio, proceso que no es exclusivo de Galicia sino que se puede generalizar al menos al conjunto de Europa. Los datos físicos del funcionamiento del metabolismo agrario gallego para el periodo, así como para el conjunto de España, no pueden ser más contundentes (figura 5). Si bien hasta la década de los sesenta del siglo XX la alimentación del ganado dependía mayoritariamente de los pastos (lo que explica la necesidad de mantener equilibrios. entre los usos del suelo y la funcionalidad de instituciones como los comunales), a partir de ese momento la alimentación ganadera depende fundamentalmente de piensos de calidad procedentes de los cultivos y de transformación industrial. A partir de los años ochenta además un porcentaje significativo de estos piensos proceden del comercio exterior por su bajo coste, que hace prácticamente 
innecesario recurrir al propio territorio para asegurar la alimentación del ganado. ${ }^{50}$ No resulta extraño por tanto que en las últimas décadas no sean las comarcas con actividad agraria las más activas sino las periurbanas como veíamos en el apartado anterior.

Figura 6 - Superficie de tierras clasificadas como comunales por provincia

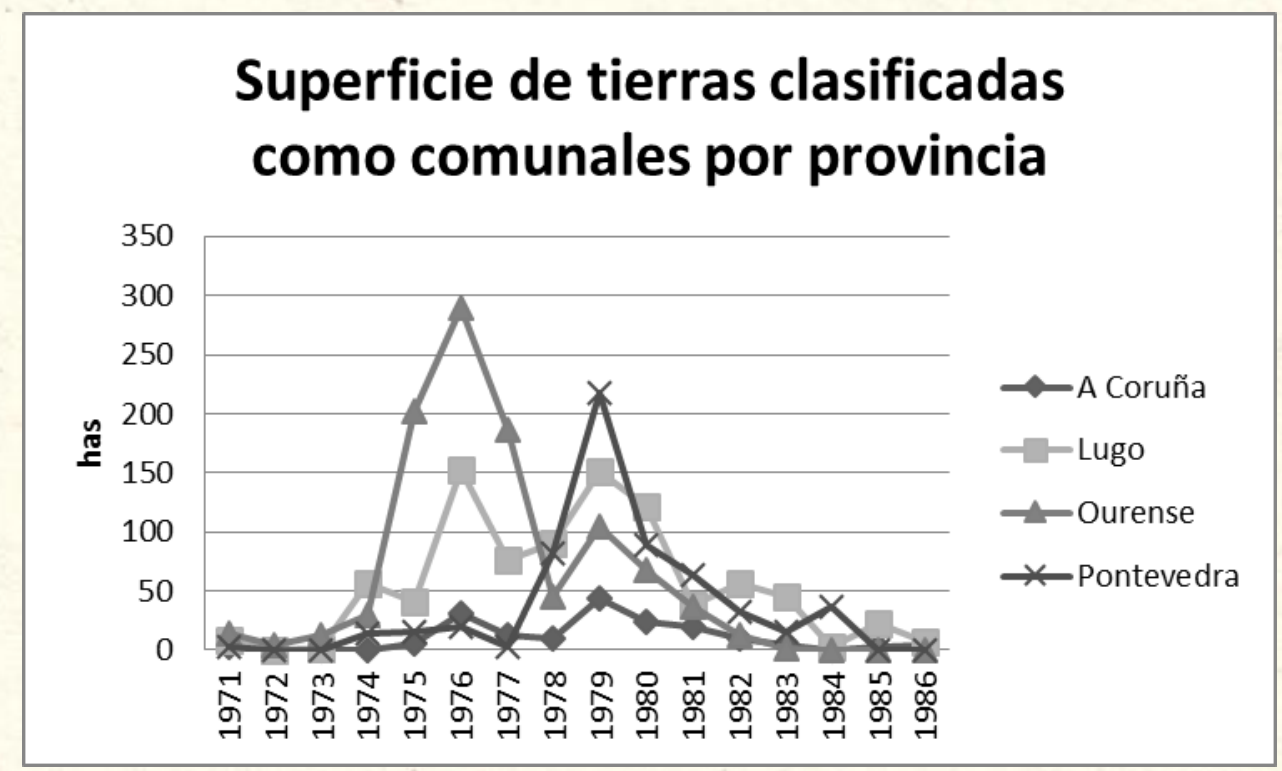

Fuente: Balboa et al, (2004)

A pesar de todo lo dicho el comunal importaba, y nada lo demuestra mejor que la conflictividad desarrollada en las décadas de los sesenta y setenta antes y después del proceso de devolución. Importaba porque en los últimos años del franquismo algunos de estos cambios que estamos describiendo apenas empezaban a tener incidencia. Importaba también por otras razones menos materiales: porque la desposesión era vista como una injusticia manifiesta, porque el monte vecinal era leído como vecinal y no como público, y porque en muchos lugares la conflictividad por la devolución del comunal irá acompañada por la lucha por la democratización a nivel local durante la transición. De hecho la ley de 1968, tal y como fue concebida por los servicios forestales que la promovieron tenía unos objetivos bastante limitados. ${ }^{51}$ Lo que esperaban de manera indisimulada los ingenieros forestales no era la devolución de todos los comunales, sino de un número significativo de ellos, especialmente concentrados en las provincias de Ourense y Lugo, que contentara a los vecinos con una participación en los beneficios de la explotación forestal y que asegurara el futuro de la repoblación forestal. Esto es lo que sucedió en los primeros años de la década de los setenta (figura 6) con la devolución a iniciativa de la administración de una significativa parte de los comunales de esas dos provincias. La devolución del comunal en muchos lugares de la provincia de Pontevedra y el sur de la provincia de A Coruña por el contrario fue posterior a la muerte del dictador en 1975 y estuvo ligada a una fuerte conflictividad en la que surgieron orgānizaciones más propias de los movimientos sociales que de las formas tradicionales de protesta campesina como la conocida como O Monte é

\footnotetext{
${ }^{50}$ David Soto, Infante-Amate, J., Guzmán, G.I., Cid, A., Aguilera, E., García, R., González de Molina, M. "The Social Metabolism of Biomass in Spain, 1900-2008: from food to feed-oriented changes in the Agro-ecosystems", Ecological Economics, 128 (2016), pp. 130-138.

${ }^{51}$ Un análisis detallado del proceso de elaboración de la ley, de los sectores implicados y de los enfrentamientos entre distintos sectores del régimen para desbordad los̄objetivos iniciales del proyecto en Balboa et al (2004; 2006). Un análisis del proceso de devolución y de los conflictos relacionados en estos dos trabajos así como en Freire (2013).
} 
Noso significativamente nombrado en lengua gallega en un contexto en el que se mezcló la reivindicación por el comunal con la lucha por la democracia, la autonomía y la lengua. ${ }^{52}$ En muchos municipios la reivindicación del comunal tuvo el apoyo cuando no fue directamente liderada por miembros de partidos de la oposición. En estos casos la devolución del comunal estuvo ligada tanto a la propiedad como al conflicto político por el poder local. No es raro encontrar ejemplos de líderes vecinales que luego ocuparon cargos políticos a nivel local en distintas formaciones políticas. ${ }^{53}$ Pero estos conflictos también tuvieron incidencia en el proceso de cambio institucional y en la configuración de la nueva comunidad descrita en el apartado anterior. En el contexto de la lucha por la democracia la idea un comunal igualitario y democráticamente gestionado tenía especial fuerza, especialmente en zonas donde los manejos tradicionales estaban en desuso o desaparecidos.

\section{Conclusiones}

Los desarrollos recientes en el análisis institucional de los comunales han empezado a llamar la atención sobre la necesidad de tener en cuenta aspectos como los ambientalesproductivos o las características de las comunidades, incluyendo en esta cuestión aspectos identitarios. Ello permite establecer diálogos fructíferos con otras tradiciones teóricas como la Economía Ecológica o la Historia Ambiental que también han prestado atención a la problemática de la pervivencia y contribución a la sustentabilidad de los comunales. En este trabajo hemos analizado un estudio de caso (la propiedad vecinal en Galicia) estudiando la situación actual y el pasado reciente desde una triple perspectiva: la evolución de los derechos de propiedad y las reglas asociadas a ellos, los cambios productivos y las mutaciones en la funcionalidad de los comunales y los aspectos identitarios que dotan de significado y dan forma a la manera en que las propias comunidades entienden su relación con el comunal. Ello nos ha permitido mostrar como parte de los comunales gallegos se han reinventado en las últimas décadas buscando nuevas interrelaciones entre comunidad, territorio y recursos. Pero al mismo tiempo la ruptura de las formas tradicionales campesinas de entender la comunidad ha conducido al abandono, la infrautilización y la degradación ambiental de buena parte de estos espacios.

\footnotetext{
${ }^{52}$ CIES, O Monte é noso. (Vigo: Galaxia, 1979).

${ }^{53}$ Balboa, et al. 2006; A. Freire, 2013.
} 